\title{
Parity violation in hydrogen revisited
}

\author{
R. W. Dunford and R. J. Holt \\ Argonne National Laboratory, Argonne, IL 60439 \\ E-mail: dunford@anl.gov
}

\begin{abstract}
We reconsider parity violation experiments in atomic hydrogen and deuterium in the light of existing tests of the Electroweak interactions, and assess whether new experiments, using improved experimental techniques, could make useful contributions to testing the Standard Model (SM). We find that, if parity experiments in hydrogen can be done, they remain highly desirable because there is negligible atomic-physics uncertainty and low energy tests of weak neutral current interactions are needed to probe for new physics beyond the SM. Of particular interest would be a measurement of the nuclear spin independent coupling $C_{1 D}$ for the deuteron at a combined error (theory + experiment) of $0.3 \%$. This would provide a factor of three improvement to the precision on $\sin ^{2} \theta_{W}$ at very low momentum transfer provided by heavy atom Atomic Parity Violation (APV) experiments. Also, experiments in $\mathrm{H}$ and $\mathrm{D}$ could provide precise measurements of three other electron-nucleon, weakneutral-current coupling constants: $C_{1 p}, C_{2 p}$, and $C_{2 D}$, which have not been accurately determined to date. Analysis of a generic APV experiment in deuterium indicates that a $0.3 \%$ measurement of $C_{1 D}$ requires development of a slow $(77 \mathrm{~K})$ metastable beam of $\approx 5 \times 10^{14} \mathrm{D}(2 \mathrm{~S}) s^{-1}$ per hyperfine component. The advent of UV radiation from free electron laser (FEL) technology could allow production of such a beam.
\end{abstract}

PACS numbers: 32.80.Ys, 11.30.Er, 12.15.Mm 


\section{Introduction}

In this report, we answer two questions concerning measurement of atomic parity violation $(\mathrm{APV})$ in hydrogen and deuterium. First, given the current level of tests of the Standard Model (SM), how well would parity violation need to be measured in hydrogen and deuterium to make a useful contribution to this body of data? Second, what experimental parameters are needed to achieve the required precision?

As to the first question, there exists a long list of impressive experimental tests of the SM [1], many involving large collaborations in high energy physics. However, as discussed in section 2, future progress may, in part, require additional high-precision, low-energy measurements[2], and in this regime, experiments in hydrogen have the advantage that the atomic structure is known to high precision and would not limit the theoretical interpretation.

Another advantage of parity experiments in hydrogen is that all of the electronnucleon, weak-neutral-current couplings can be determined by performing measurements at several level crossings in both $\mathrm{H}$ and $\mathrm{D}$. This point is discussed in Section 3 where we present updated SM predictions for the parity violation mixings at the various crossings. A summary of the results of former PNC measurements in hydrogen is presented in Section 4, and in Sections 5, and 6] we consider our question concerning the experimental requirements by evaluating a generic APV experiment in deuterium. Finally, we summarize our conclusions in section 17 .

\section{Theoretical Motivation}

Testing the SM of the electroweak interaction remains an important task of experimental physics. This effort has reached the level of precision measurements. For example, the

weak mixing angle $\sin ^{2} \theta_{W}$ has been determined to $0.06 \%$ at the $\mathrm{Z}$ boson mass. Most of the data confirming the SM have been obtained at high energy near the $\mathrm{Z}$ resonance. The excellent agreement at the Z-pole was obtained by the weighted average of two experiments that disagree with one another by $3.4 \sigma$. Moreover, there have been recent measurements at lower energy, such as the muon g-2 experiment [3], the NuTeV neutrino deep inelastic scattering experiment [4, and the SLAC E-158 polarized Møller Scattering experiment [5]. In the muon g-factor experiment there is a 3.4 standard deviation difference between the experimental result and the theoretical calculation [6] suggesting new physics beyond the Standard Model. This situation has motivated additional low energy measurements of the electroweak interactions, such as the Qweak [7, 8] and DIS-Parity [9, 10] experiments at the Thomas Jefferson National Accelerator Facility (JLab).

A useful way to assess the low energy tests of the electroweak interaction is to consider the predicted running of weak mixing angle $\sin ^{2} \theta_{W}$. The SM predicts that this parameter evolves as a function of momentum transfer [2, 11, 12]. This evolution can be checked by performing precision low energy experiments and comparing the results with 
precise measurements at high energy. In this respect, tests done near zero momentum transfer such as APV or low energy electron scattering experiments are ideal. In the $\overline{M S}$ scheme, Erler and Ramsey-Musolf [2, 12] find for the value of the weak mixing angle at zero momentum transfer:

$$
\sin ^{2} \hat{\theta}_{W}(0)=0.23867 \pm 0.00016 .
$$

In the same scheme the value at the Z-pole is [1]:

$$
\sin ^{2} \hat{\theta}_{W}\left(M_{Z}\right)=0.23122 \pm 0.00015,
$$

so that the zero momentum transfer value is larger by about 3\%. Several low energy measurements test this prediction. APV (Cs[13] and Tl[14, 15]) establishes the running of weak mixing angle with a $0.9 \%$ measurement of $\sin ^{2} \hat{\theta}_{W}$ that is one standard deviation below the SM prediction. The E-158 Møller scattering experiment has an error of $0.6 \%$ which is one sigma above the SM. NuTeV, a deep inelastic neutrino scattering experiment, reports a $3 \sigma$ discrepancy with the SM value.

The Qweak experiment aims to improve the limits on the semi-leptonic weak neutral current couplings by measuring the weak charge of the proton $\mathrm{Q}_{W}(\mathrm{p})$. In lowest order the weak charge is given by [16]:

$$
Q_{W}(p)=\left(1-4 \sin ^{2} \theta_{W}\right) .
$$

Since $\sin ^{2} \theta_{W}$ is close to $1 / 4, Q_{W}(p)$ is suppressed and particularly sensitive to higher order corrections and new physics beyond the SM.

To be more precise, we can use the SM expressions for the electron-hadron processes from Ref. [1, including radiative corrections, to derive the relation between the uncertainties in the weak charge $Q_{W}(Z, N)$ and $\sin ^{2} \hat{\theta}_{W}$ for an atom with nuclear charge $Z$ and neutron number $N$. We find:

$$
\frac{\Delta\left(\sin ^{2} \theta_{W}\right)}{\sin ^{2} \theta_{W}}=\left(-0.078+1.0785 \frac{N}{Z}\right) \frac{\Delta Q_{W}}{Q_{W}} .
$$

For $\mathrm{H}, \mathrm{D}$, and Cs the factors multiplying $\Delta Q_{W} / Q_{W}$ are $-0.078,1.00$, and 1.45 , respectively. For example, a measurement of $Q_{W}(p)$ with a precision of $4 \%$ determines $\sin ^{2} \theta_{W}$ to $0.3 \%$. For the Boulder Cs experiment, if the experimental error $(\sim 0.35 \%)$ [13] and atomic theory error $(\sim 0.5 \%)$ are combined in quadrature, the total uncertainty in $Q_{W}(C s)$ is $\sim 0.6 \%$ which corresponds to an uncertainty of $\sim 0.9 \%$ in $\sin ^{2} \theta_{W}$ based on Eq. 4, and this is the dominant contribution to the current APV limit on $\sin ^{2} \theta_{W}$. For deuterium, Eq. 4 indicates that the fractional uncertainty in $Q_{W}(D)$ is equal to the fractional uncertainty in $\sin ^{2} \theta_{W}$.

The need for precision measurements of semileptonic weak neutral current couplings at low momentum transfer obliges a renewed consideration of measurements in hydrogen and deuterium where precision is not limited by calculational uncertainties. In this respect, both the heavy atom APV experiments and polarized electron scattering experiments are susceptible to hadronic or atomic effects. Qweak aims to measure the parity violating elastic electron-proton scattering asymmetry at $Q^{2} \approx 0.03 \mathrm{GeV}^{2}$. This 
process is proportional to the sum of $Q_{W}(\mathrm{p})$ and a proton form factor $F^{p}\left(Q^{2}, \theta\right)$. The form factor contribution must be subtracted from the scattering asymmetry in order to extract $Q_{W}(\mathrm{p})$. In principle, this extrapolation would require a hadronic structure calculation, but an extensive series of parity violating electron-proton scattering experiments at higher $Q^{2}$ will allow extrapolation to $Q^{2}=0$ with an uncertainty of about $2 \%$ in the weak charge[2]. The heavy atom APV experiments require sophisticated atomic theory calculations in order to extract the weak charge from the experimental results. In the case of $\mathrm{Cs}$, the atomic structure uncertainty is currently about $0.5 \%$. To go beyond this in heavy atoms probably requires measuring APV for a series of isotopes of an atom and forming ratios of the results. These ratios determine $Q_{W}$ and are less sensitive to calculational uncertainties. However, this procedure is subject to other uncertainties arising from variations in neutron distributions along the chain, which will limit the ultimate precision[2].

Apart from the potential of a precision measurement of $\sin ^{2} \theta_{W}$ and sensitivity to new physics, experiments in hydrogen and deuterium provide the opportunity to determine all four of the electron-nucleon, weak-neutral-current coupling constants: $C_{1 p}$, $C_{2 p}, C_{1 n}$, and $C_{2 n}$ [17, 18]. Here $C_{1 p}$ and $C_{1 n}$ are the nuclear spin independent couplings for the proton and neutron. (Note that $Q_{W}(p) \equiv 2 C_{1 p}\left[16,[18]\right.$.) $C_{2 p}$ and $C_{2 n}$ are the nuclear spin dependent couplings. These four parameters are important because they are basic properties of the neutron and proton. The constants for the neutron can be obtained by comparing results from hydrogen and deuterium experiments. For deuterium we use the notation [19]:

$$
\begin{aligned}
& C_{1 D}=C_{1 p}+C_{1 n} \\
& C_{2 D}=C_{2 p}+C_{2 n} .
\end{aligned}
$$

In terms of the quark coupling constants $C_{1 u}, C_{1 d}, C_{2 u}$ and $C_{2 d}$ (Ref. [1]), the proton and deuteron couplings have the values[20, 21, 22, 23]:

$$
\begin{aligned}
& C_{1 p}=-\left(2 C_{1 u}+C_{1 d}\right) \\
& C_{1 D}=-3\left(C_{1 u}+C_{1 d}\right) \\
& C_{2 p}=-0.935 C_{2 u}+0.360 C_{2 d} \\
& C_{2 D}=-0.45\left(1.1 C_{2 u}+0.9 C_{2 d}\right) .
\end{aligned}
$$

The constants $C_{1 p}$ and $C_{1 D}$ can be determined from APV experiments with little strong interaction uncertainty. By contrast, $C_{2 p}$ and $C_{2 D}$ involve considerable strong interaction uncertainty, as has been discussed by a number of authors [22, 24, 25].

In Table 1 we give experimental and theoretical values for these couplings. The experimental numbers in column 2 are based on a "model-independent fit" to all neutral current parameters which are presented in Table 10.8 of Ref. [1]. The fit allowed for an arbitrary electroweak gauge theory. The final column of Table1 gives the SM predictions based on Eq. 6, Table 10.3 of Ref. [1], and $\sin ^{2} \hat{\theta}_{W}=0.23122$. Theoretical corrections from the anapole [26] and strange quark [27] contributions are not included. All of the couplings except $C_{1 D}$ are suppressed in the Standard Model. For $C_{1 p}$ and $C_{2 p}$ the 
Table 1. Parity violating weak neutral current couplings for H and D.

\begin{tabular}{llc}
\hline Coupling & From model-independent fit [1] & Standard Model \\
\hline$C_{1 p}$ & $0.082 \pm 0.034$ & 0.0355 \\
$C_{1 D}$ & $-0.441 \pm 0.012$ & -0.4587 \\
$C_{2 p}$ & $-0.16 \pm 0.26$ & 0.043 \\
$C_{2 D}$ & $-0.32 \pm 0.4$ & 0.007 \\
\hline
\end{tabular}

suppression arises because they are proportional to $1-4 \sin ^{2} \theta_{W}$ in lowest order. The coupling $C_{2 D}$ vanishes exactly in lowest order and the small residual value is entirely due to higher order corrections, so this parameter is quite sensitive to these corrections. Although $C_{1 D}$ has been determined (indirectly) to about $3 \%$, the uncertainty in $C_{1 p}$ is comparable to the size of the SM prediction, and both $C_{2 p}$ and $C_{2 D}$ are undetermined.

An attractive goal for a future APV experiment would be a $0.3 \%$ measurement of the nuclear spin independent coupling for the deuteron $C_{1 D}$. This would determine $\sin ^{2} \hat{\theta}_{W}(0)$ with a combined error (theoretical + experimental) of $0.3 \%$ which is a factor of three improvement on the limit provided by the heavy atom APV experiments. This is comparable to the limit on $\sin ^{2} \hat{\theta}_{W}(0)$ expected from the Qweak experiment, but would provide complementary information on the isospin dependence of the e-nucleon electroweak couplings [28].

A measurement of the nuclear spin independent coupling in hydrogen at the same level of absolute precision would provide a $4 \%$ measurement of $C_{1 p}$, which together with a measurement of $C_{1 D}$ would provide a constraint on the isospin dependence of the nuclear-spin-independent weak-neutral-current interaction with one experimental setup. For $C_{2 p}$ and $C_{2 D}$, measurements with absolute precisions similar to a $0.3 \%$ measurement of $C_{1 D}$ could provide the first determinations of these quantities with an error of $3 \%$ for $C_{2 p}$ and $20 \%$ for $C_{2 D}$. Again, the isospin dependence of the nuclear spin dependent couplings could be constrained by comparing the two measurements.

\section{Parity mixing in hydrogen and deuterium}

In planning parity experiments in hydrogen, it is natural to take advantage of the metastable $2^{2} \mathrm{~S}_{1 / 2}$ state. Metastable hydrogen atoms live long enough $(\sim 122 \mathrm{~ms})$ to survive the length of a typical laboratory atomic beam apparatus, and because of the near degeneracy of the $2^{2} \mathrm{~S}_{1 / 2}$ and $2^{2} \mathrm{P}_{1 / 2}$ states, level crossings occur at relatively modest magnetic fields $(\sim \mathrm{kG})$, and this provides a means for separately determining the weak interaction coupling constants, since different combinations of constants are resonantly enhanced at the various level crossings. In order to determine all four coupling constants in Table 1, it is necessary to perform experiments at several magnetic field settings in both $\mathrm{H}$ and $\mathrm{D}$, and combine the results to isolate the separate constants. In this section, we review the parity mixing matrix elements at the various crossings in $\mathrm{H}$ and $\mathrm{D}$ and 


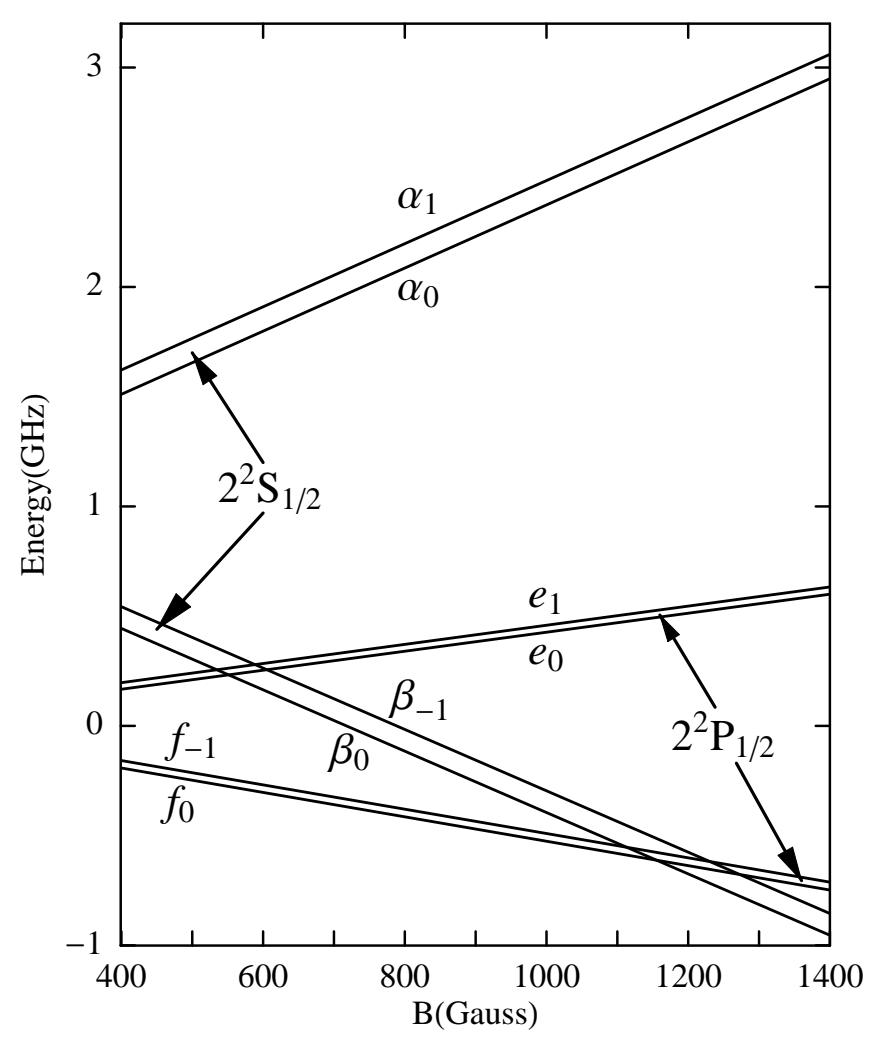

Figure 1. Zeeman diagram showing the $2^{2} \mathrm{~S}_{1 / 2}$ and $2^{2} \mathrm{P}_{1 / 2}$ levels in hydrogen in a static magnetic field[29]. The states are labeled using Lamb's nomenclature [30]. Subscripts give the values of the good quantum number $m_{F}$.

present updated SM predictions for their strengths.

Fig. 11 shows the relevant energy levels of hydrogen in a static magnetic field of 400-1400 G. In this region, the Zeeman energy from the coupling to the magnetic field is much less than the fine structure splitting so the total electronic angular momentum $\mathrm{J}$ is still a good quantum number. On the other hand, at fields above $\sim 200 \mathrm{G}$, the electron and nuclear spins are decoupled so the natural basis is $\left|\mathrm{Jm}_{\mathrm{J}} \mathrm{Im}_{\mathrm{I}}\right\rangle$, where $\mathrm{I}$ is the nuclear spin. In Fig 1 the $2{ }^{2} \mathrm{~S}_{1 / 2}$ levels are labeled $\alpha\left(\mathrm{m}_{\mathrm{J}}=+1 / 2\right)$ and $\beta\left(\mathrm{m}_{\mathrm{J}}=-1 / 2\right)$, while the $2^{2} \mathrm{P}_{1 / 2}$ states are labeled e $\left(\mathrm{m}_{\mathrm{J}}=+1 / 2\right)$ and $\mathrm{f}\left(\mathrm{m}_{\mathrm{J}}=-1 / 2\right)$ [31]. The subscripts are $\mathrm{m}_{\mathrm{F}}=\mathrm{m}_{\mathrm{J}}+\mathrm{m}_{\mathrm{I}}$.

Fig. 2 shows blowups of the $\beta$ e level crossings in hydrogen and deuterium. The selection rule for $\mathrm{H}_{\mathrm{PNC}}$ is $\Delta \mathrm{m}_{\mathrm{F}}=0$, so only the levels $\beta_{0}$ and $\mathrm{e}_{0}$ in hydrogen are mixed. The value of the matrix element is given in Table 2, which indicates that a measurement at the $\beta$ e crossings in hydrogen is sensitive only to the nuclear spin dependent coupling $\mathrm{C}_{2 p}$. In deuterium, parity mixing is resonant at two $\beta$ e crossings which, as indicated in Table 2, are sensitive only to $C_{2 D}$.

Fig. 3 shows the $\beta \mathrm{f}$ level crossings in hydrogen and deuterium. Parity mixing is resonant at two crossings in hydrogen. Table 2 indicates the matrix elements depend 


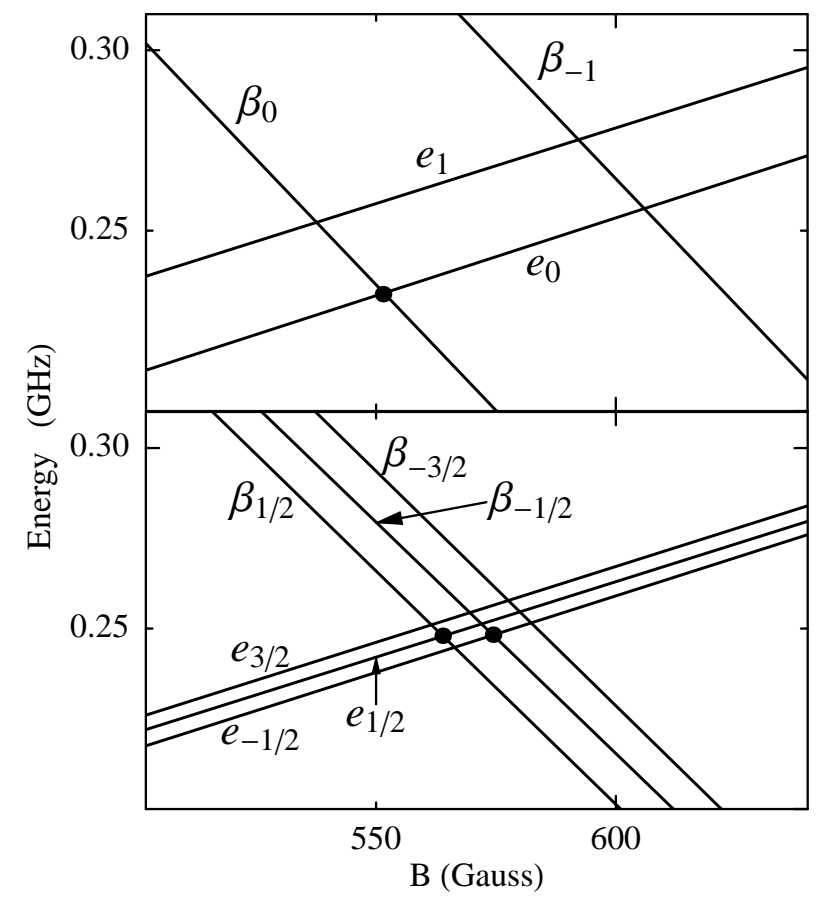

Figure 2. Parity mixings resonant at the $\beta e$ crossings in hydrogen (upper part) and deuterium (lower part).

on nearly orthogonal combinations of $C_{1 p}$ and $C_{2 p}$. So parity experiments designed to use the $\beta \mathrm{f}$ crossings in hydrogen provide a means to measure both of these constants. For the $\beta \mathrm{f}$ crossings in deuterium, there are three crossings of levels mixed by the weak interaction. From Table 2 it is seen that two of these depend on orthogonal combinations of $C_{1 D}$ and $C_{2 D}$ while the crossing of $f_{-1 / 2}$ and $\beta_{-1 / 2}$ is, to good approximation, sensitive only to $C_{1 D}$.

\section{Current limits on parity violation in hydrogen}

There have been numerous suggestions for APV experiments in hydrogen. One possibility is to observe PNC asymmetries in the single photon decay $2 \mathrm{~S}_{1 / 2} \rightarrow 1 \mathrm{~S}_{1 / 2}$ $+\gamma$ of a metastable beam in vacuum[17], or in a beam perturbed by applied electric and magnetic fields [32, 33]. These ideas have not led to practical experiments in hydrogen although they hold promise for experiments in heavy one-electron ions [33]. For hydrogen, the most promising approach is to drive optical or microwave transitions $2 \mathrm{~S}_{1 / 2}$ $\rightarrow \mathrm{nS}_{1 / 2}$ and look for a pseudoscalar dependence on the transition rate [34, 35, 36, 37, 38]. Microwave (or $\mathrm{RF}[39]$ ) transitions $2 \mathrm{~s} \rightarrow 2 \mathrm{~s}$ ' within $\mathrm{n}=2$ are particularly attractive because Doppler broadening is negligible, and linewidths can be small. Also, relatively high power is readily available and the interaction volume can be large. The possibility for narrow linewidths is particularly significant since it allows complete separation of the various hyperfine transitions, and, as discussed in Section 5, a narrow line is required 


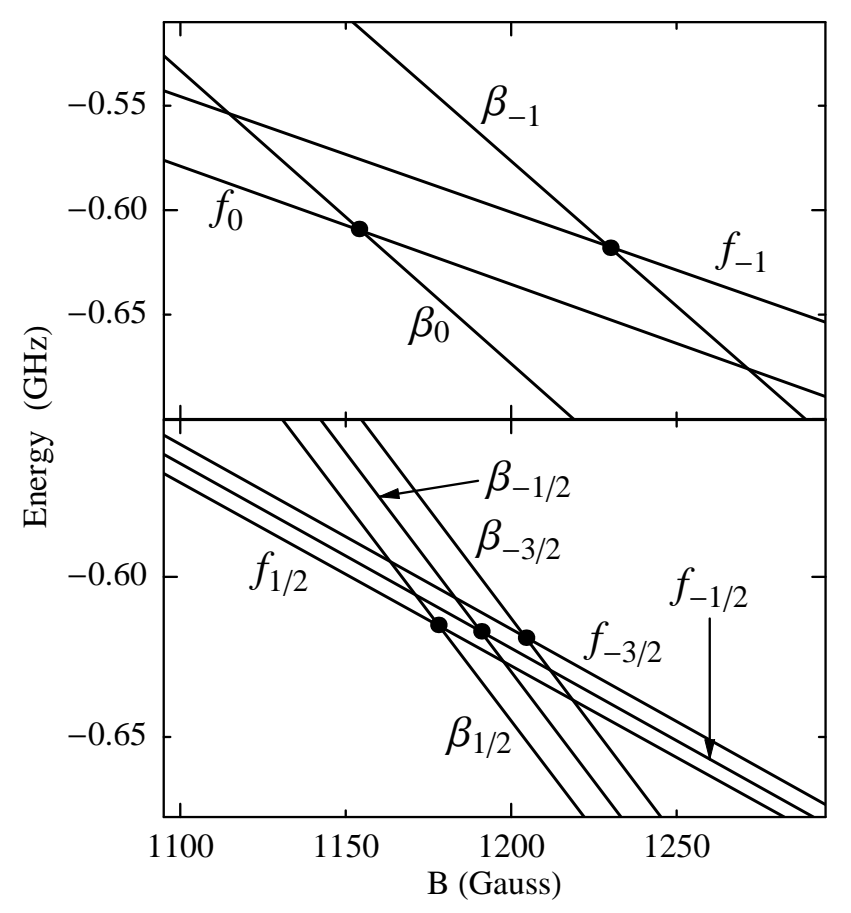

Figure 3. Parity mixings resonant at the $\beta f$ crossings in hydrogen (upper part) and deuterium (lower part).

Table 2. Matrix elements for parity-violating, weak-neutral-current couplings for hydrogen and deuterium at the level crossings. In units of $\mathrm{i} \overline{\mathcal{V}}_{\mathrm{w}} \approx 2 \pi \times 0.013 \mathrm{~s}^{-1}$. $C_{1 D} \equiv C_{1 p}+C_{1 n}$ and $C_{2 D} \equiv C_{2 p}+C_{2 n}$.

\begin{tabular}{lcc}
\hline Matrix Element & Value & Standard Model \\
\hline Hydrogen & & \\
$\left\langle e_{0}|V| \beta_{0}\right\rangle$ & $-2 C_{2 p}$ & -0.086 \\
$\left\langle f_{0}|V| \beta_{0}\right\rangle$ & $C_{1 p}+1.1 C_{2 p}$ & 0.083 \\
$\left\langle f_{-1}|V| \beta_{-1}\right\rangle$ & $C_{1 p}-C_{2 p}$ & -0.007 \\
Deuterium & & \\
$\left\langle e_{1 / 2}|V| \beta_{1 / 2}\right\rangle$ & $-\sqrt{2} C_{2 D}$ & -0.010 \\
$\left\langle e_{-1 / 2}|V| \beta_{-1 / 2}\right\rangle$ & $-\sqrt{2} C_{2 D}$ & -0.010 \\
$\left\langle f_{1 / 2}|V| \beta_{1 / 2}\right\rangle$ & $C_{1 D}+C_{2 D}$ & -0.452 \\
$\left\langle f_{-1 / 2}|V| \beta_{-1 / 2}\right\rangle$ & $C_{1 D}$ & -0.459 \\
$\left\langle f_{-3 / 2}|V| \beta_{-3 / 2}\right\rangle$ & $C_{1 D}-C_{2 D}$ & -0.466 \\
\hline
\end{tabular}


to optimize the sensitivity to parity violation.

Starting in the late 1970s, hydrogen parity experiments were performed at the University of Michigan [34, 36, 40, 41, 44], The University of Washington [38, 45], and Yale University [35, 39]. The experiments were motivated in part by the near degeneracy of the $2^{2} \mathrm{~S}_{1 / 2}$ and $2^{2} \mathrm{P}_{1 / 2}$ levels in hydrogen which enhances the parity mixing and provides an opportunity for detection of PNC effects even though hydrogen does not benefit from the $\mathrm{Z}^{3}$ enhancement as a function of nuclear charge which motivates parity experiments in heavy atoms [46].

All of the hydrogen APV experiments were based on fast $(\sim 500 \mathrm{eV})$ beams of metastable hydrogen atoms and aimed to measure $C_{2 p}$. Typically, the beam was prepared in one of the $\alpha$ hyperfine states and a transition $\alpha \rightarrow \beta$ to one of the $\beta$ hyperfine states was driven. The transition was observed by selective quenching of the resulting $\beta$ state atoms and detecting the Lyman- $\alpha$ radiation emitted.

The transitions were driven by a parity conserving amplitude $\mathrm{A}_{\mathrm{PC}}$ and a parity nonconserving amplitude $A_{P N C}$. The parity nonconserving amplitude proceeds via an intermediate $2 P_{1 / 2}$ state:

$$
\alpha \stackrel{\epsilon^{\omega}}{\longrightarrow} 2 P_{1 / 2} \stackrel{\mathrm{V}_{\mathrm{w}}}{\longrightarrow} \beta,
$$

where the first step is an $E 1$ transition driven by microwave electric field $\epsilon^{\omega}$, and the second step is the $2^{2} \mathrm{~S}_{1 / 2}-2^{2} \mathrm{P}_{1 / 2}$ coupling by the weak interaction. This is also called a "weak induced amplitude".

Two types of parity conserving amplitudes $\mathrm{A}_{\mathrm{PC}}$ were used. One of these was an M1 amplitude[35]:

$$
\alpha \stackrel{b^{\omega}}{\longrightarrow} \beta
$$

where $b^{\omega}$ is the microwave magnetic field. More commonly a "Stark induced amplitude" was used: [36, 38]:

$$
\alpha \stackrel{\epsilon^{\omega}}{\longrightarrow} 2^{2} \mathrm{P}_{1 / 2} \stackrel{E^{\mathrm{S}}}{\longrightarrow} \beta
$$

here, the first step is a microwave $E 1$ coupling to an intermediate $2^{2} \mathrm{P}_{1 / 2}$ state driven by $\epsilon^{\omega}$, and the second step is a $2^{2} \mathrm{~S}_{1 / 2}-2^{2} \mathrm{P}_{1 / 2}$ coupling by a static electric field $E^{s}$.

The experiments searched for a parity violating (pseudoscalar) interference term between $\mathrm{A}_{\mathrm{PNC}}$ and $\mathrm{A}_{\mathrm{PC}}$ in the transition rate, that changed sign as the "handedness" of the interaction region was switched. The main differences among the various schemes were in the configurations of fields in the interaction regions, and the pseudoscalars of interest. Particularly significant is that in the Michigan experiment, $\mathrm{A}_{\mathrm{PC}}$ and $\mathrm{A}_{\mathrm{PNC}}$ were driven simultaneously in a single microwave cavity, while in the Yale and U. Washington experiments, separate oscillating field regions were used for $A_{\mathrm{PNC}}$ and $\mathrm{A}_{\mathrm{PC}}$.

These experiments were not able to observe parity violation (for a short review see [47]). The best limit was obtained by Fehrenbach [44] who reported:

$$
C_{2 p}=1.5 \pm 1.5 \pm 22 .
$$


Here, the first error is statistical and the second is systematic. From Table 1, we see that the value expected based on the Standard model is $\mathrm{C}_{2 p}=0.036$. So the statistical error is a factor of 40 larger than the expected effect while the systematic error is 600 times larger than the expected effect. It is clear that more than an incremental improvement is necessary if a useful measurement is to be obtained. Some ideas of how this might be accomplished are given in sections 5 and 6.

\section{Sensitivity of a generic deuterium APV experiment}

The original hydrogen parity experiments could not have observed PNC. The problems were a lack of sufficient sensitivity and large systematic errors. In the next two sections we discuss a particular idea for an APV experiment in deuterium aimed at providing a $0.3 \%$ measurement of $\mathrm{C}_{1 D}$. The conclusions of this paper do not rest on this particular scheme, as there are many other possibilities which would have roughly the same sensitivity. Rather, we chose this example after considering some general guidelines for a future H/D parity experiment. First, in comparison to the original H APV experiments, the signal-to-noise-ratio $\mathrm{S} / \mathrm{N}$ has to be improved by more than two orders of magnitude. Then, to improve handling of systematic effects, it is important to have precise control over the alignment of the apparatus and all applied fields. To make this less daunting, the apparatus should be simple - a few homogeneous applied fields is preferred over more complicated configurations. Perturbations of the beam due to surfaces (apertures, walls, etc.) should be minimized, and all surfaces should be kept as far from the beam as possible. The apparatus should be "clean" i.e. surfaces should be carefully prepared to minimize stray electric fields, and ultra-high vacuum techniques should be used. The beam density should be as low as possible consistent with the needed intensity and there should be no charged particles, or field configurations which tend to trap charged particles.

One can increase $\mathrm{S} / \mathrm{N}$ by developing a more intense metastable beam. In addition, there are advantages in both sensitivity, and in reducing systematic errors to using a slow beam 38, 41]. In Appendix B we discuss the requirements for producing a slow $(77 \mathrm{~K})$ metastable beam with about $5 \times 10^{14} \mathrm{D}(2 \mathrm{~s}) \mathrm{s}^{-1}$ per hyperfine level. An important consideration is that there is a limit on the density of metastable atoms in the beam due to quenching by beam-beam collisions. This is discussed in Appendix A.

A particularly simple configuration for the interaction region would involve driving an M1 transition [35] with a plane polarized microwave beam incident at right angles to the metastable beam. This would eliminate the need for an additional applied electric field to drive $A_{\mathrm{PC}}$. Also, eliminating the microwave cavity (or cavities), has some advantages. The standing wave pattern in a cavity leads to spatial variations in the fields which can complicate the transition amplitudes [48] and make analyzing systematic errors more difficult. In addition, apertures at the entrance or exit to the cavity perturb both the atoms and the cavity modes.

A practical way to realize this is to use a microwave-open-ring-resonator [49, 50, 51] 


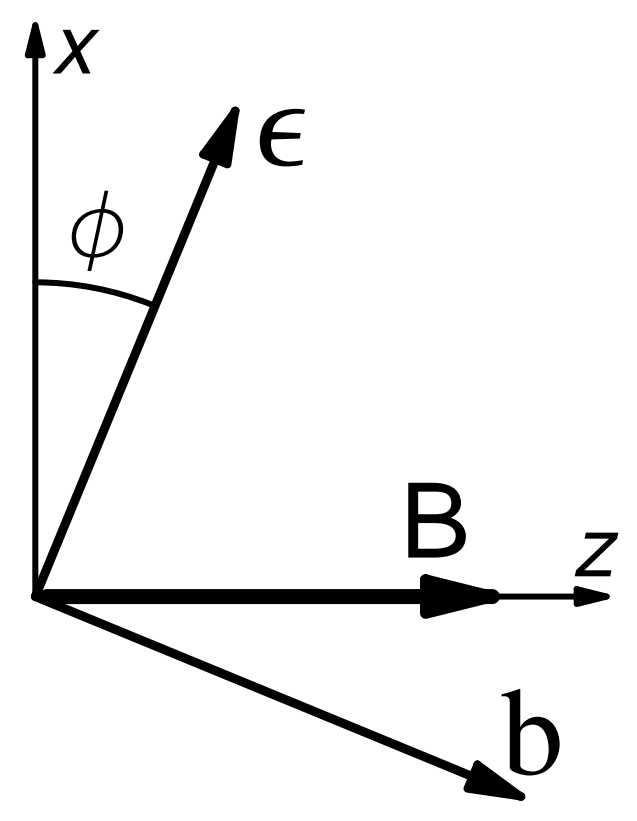

Figure 4. Fields in the interaction region. The metastable $\mathrm{D}$ beam is travelling along the $\mathrm{z}$ axis. The microwave electric $\boldsymbol{\epsilon}$ and magnetic $\mathbf{b}$ fields lie in the $\mathrm{x}-\mathrm{z}$ plane. $\mathbf{B}$ is a static magnetic field.

in the interaction region. Complete control of the polarization in such a resonator is possible using quasi-optical techniques[52]. Such a resonator allows the propagation direction of the traveling waves to be switched, providing the ability to reverse the microwave propagation vector $\mathbf{k}$.

As an example, we consider a measurement of $C_{1 D}$ at the crossing of the levels $f_{-1 / 2}$ and $\beta_{-1 / 2}$, utilizing the configuration of fields shown in Fig. 4. The $\mathrm{D}(2 \mathrm{~s})$ beam is directed along the $\mathrm{z}$ axis, collinear with a static magnetic field $\mathbf{B}$. The beam of microwaves propagates into the page $(\hat{\mathbf{k}}=-\hat{\mathbf{y}})$ and the frequency is set to drive the M1 transition $\alpha_{+1 / 2} \rightarrow \beta_{-1 / 2}$. The microwave electric polarization vector $\boldsymbol{\epsilon}$ lies in the $\mathrm{x}-\mathrm{z}$ plane, and is orthogonal to the microwave magnetic field $\mathbf{b}$. The vector $\boldsymbol{\epsilon}$ is a combination of fields $\boldsymbol{\epsilon}_{x}$ and $\boldsymbol{\epsilon}_{z}$ [52]. These fields are derived from the same source but have a phase difference of $\theta$ and separately controlled amplitudes. The resulting field is given by:

$$
\boldsymbol{\epsilon}(t)=\boldsymbol{\epsilon}_{x} \cos (\nu t)+\boldsymbol{\epsilon}_{z} \cos (\nu t+\theta) .
$$

Here, we will set $\theta=0$ or $\pi$, in which case, the microwave polarization is linear. The polarization angle $\phi$ relative to the $x$ axis is determined by the relative strength of $\boldsymbol{\epsilon}_{x}$ and $\boldsymbol{\epsilon}_{z}$. When $\theta$ is changed from 0 to $\pi, \phi$ goes to $-\phi$. Other values of $\theta$ give circular or elliptical polarization. The amplitudes in the experiment are shown schematically in Fig 5. The parity conserving part $\mathrm{A}_{\mathrm{PC}}$ is an $M 1$ amplitude driven by the component of the microwave magnetic field along the $x$ axis $\left(b_{x}\right)$.

The parity nonconserving amplitude $\mathrm{A}_{\mathrm{PNC}}$ proceeds via the intermediate level $f_{-1 / 2}$ and involves the weak interaction $\mathrm{H}_{\mathrm{PNC}}$ and an $E 1$ coupling via the microwave 


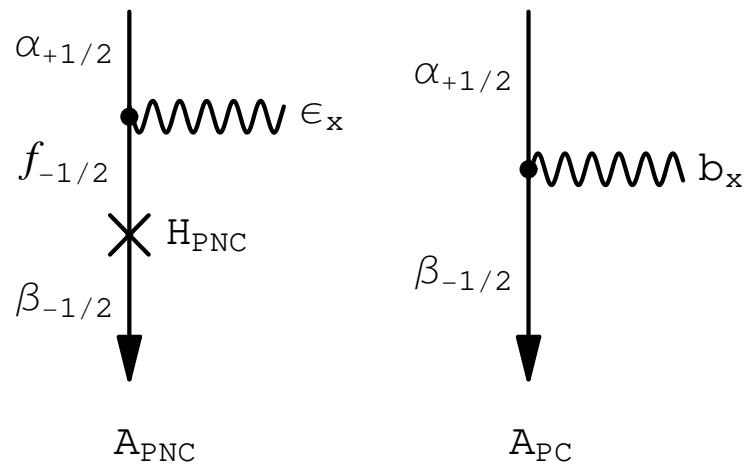

Figure 5. Parity conserving $A_{P C}$ and nonconserving $A_{P N C}$ amplitudes.

electric field component along the $x$ axis $\left(\epsilon_{x}\right)$. The analysis of this experiment requires consideration of the equations of motion for the three levels $\alpha_{+1 / 2}, \beta_{-1 / 2}$, and $f_{-1 / 2}$ but, to sufficient approximation for the present considerations, can be reduced to an effective two-level problem by introducing the parameter $\gamma_{\alpha_{+1 / 2}}$ which is the decay rate of the $\alpha_{+1 / 2}$ level due to the quenching $\alpha_{+1 / 2} \rightarrow f_{-1 / 2}$ by the microwaves [41]. The amplitudes $a$ for the $\alpha_{+1 / 2}$ state and $b$ for the $\beta_{-1 / 2}$ state obey the equations:

$$
\begin{aligned}
& \mathrm{i} \dot{a}=\Lambda^{\dagger} \exp \left[-\mathrm{i}\left(\omega_{\alpha_{+1 / 2} \beta_{-1 / 2}}-\nu\right) t\right] b-\frac{1}{2} \mathrm{i} \gamma_{\alpha_{+1 / 2}} a \\
& \mathrm{i} \dot{b}=\Lambda \exp \left[+\mathrm{i}\left(\omega_{\alpha_{+1 / 2} \beta_{-1 / 2}}-\nu\right) t\right] a
\end{aligned}
$$

where[53]:

$$
2 \hbar \Lambda=-\mu_{0} \mathrm{~b}_{x}-\mathrm{i} \overline{\mathcal{V}}_{\mathrm{w}} \mathrm{C}_{1 \mathrm{D}} d_{2} \epsilon_{x} \Delta_{\beta_{-1 / 2} \mathrm{f}_{-1 / 2}} .
$$

The parameter $\overline{\mathcal{V}}_{\mathrm{w}}=2 \pi(0.013) s^{-1}$ gives the magnitude of the weak interaction matrix element, and $d_{2}=\sqrt{3} e a_{0}$. The parameter $\Delta_{\beta_{-1 / 2} f_{-1 / 2}}$ is defined by:

$$
\Delta_{i j}=\frac{1}{\omega_{i j}+\mathrm{i} \gamma_{2 p} / 2} .
$$

Here, $\omega_{i j} \equiv \omega_{i}-\omega_{j}$, and $\gamma_{2 p}=2 \pi \times 10^{8} s^{-1}$ is the natural decay rate of the $2 p$ state. The beam is prepared in the $\alpha_{+1 / 2}$ state so the initial conditions are:

$$
\begin{aligned}
& a(0)=1 \\
& b(0)=0 .
\end{aligned}
$$

For simplicity, we treat the case of exact resonance $\omega_{\alpha_{+1 / 2} \beta_{-1 / 2}}-\nu=0$, and also choose the maximum interference between the two terms in Eq. 13 which requires $\omega_{\beta_{-1 / 2} f_{-1 / 2}}=0$, i.e. that the magnetic field be tuned to the center of the level crossing. If the atom spends a time $\tau$ in the interaction region, the amplitude to be in the state $\beta_{-1 / 2}$ after this time is given by:

$$
b(\tau)=-\mathrm{i} \Lambda \frac{1-\exp \left(-\gamma_{\alpha_{+1 / 2}} \tau / 2\right)}{\gamma_{\alpha_{+1 / 2}} / 2} .
$$

The two terms in $\Lambda$ (Eq. 13) determine the two amplitudes of interest. $A_{P C}$ is proportional to the first term while $A_{P N C}$ is proportional to the second. Since the 
Table 3. Parameters determining the optimized sensitivity (Eq. 221)

\begin{tabular}{cll}
\hline Symbol & Meaning & Assumed for Figs. [6 and 7 \\
\hline$\eta$ & Total detection efficiency & 0.5 \\
$J$ & Metastable flux/hyperfine component & - \\
$T$ & Counting time & 5 days \\
$\tau$ & Interaction time & - \\
$\mathrm{i} \overline{\mathcal{V}}_{\mathrm{w}}$ & H PNC coupling strength $(\mathrm{n}=2)$ & $2 \pi \times 0.013 \mathrm{~s}^{-1}$ \\
$\gamma_{2 p}$ & 2p decay rate & $2 \pi \times 10^{8} \mathrm{~s}^{-1}$ \\
\hline
\end{tabular}

electric $\boldsymbol{\epsilon}$ and magnetic $\mathbf{b}$ fields in the plane wave are orthogonal, $\mathrm{b}_{x}=\mathrm{b} \sin \phi$ and $\epsilon_{x}=\epsilon \cos \phi$, so the sign of the interference term changes as $\phi \rightarrow-\phi$. The interference term is proportional to the pseudoscalar $(\boldsymbol{\epsilon} \cdot \mathbf{B})(\boldsymbol{\epsilon} \times \mathbf{k}) \cdot \mathbf{B}$, so it also changes sign if the microwave propagation vector is reversed, i.e. $\mathbf{k} \rightarrow-\mathbf{k}$; but it is even under a reversal of $\mathbf{B}$. This pseudoscalar can appear in a T-even theory due to the presence of damping of the $2 \mathrm{p}$ state [37, 42, 43].

The parameter $\gamma_{\alpha_{+1 / 2}}$ is determined in a separate calculation. If the magnetic field is set at the level crossing and the microwave frequency is set at the center of the $\alpha_{+1 / 2} \rightarrow \beta_{-1 / 2}$ resonance we have[30]:

$$
\gamma_{\alpha_{+1 / 2}}=\gamma_{2 p}\left(\frac{d_{2} \epsilon_{x}}{\hbar \gamma_{2 p}}\right)^{2}
$$

Two quantities are useful in assessing the sensitivity of this type of APV experiment. The first is the fraction of the rate that is due to the PNC interference term, which we will call the asymmetry $\mathcal{A}$ :

$$
\begin{aligned}
\mathcal{A} & =4 \frac{\overline{\mathcal{V}}_{\mathrm{w}}}{\gamma_{2 p}} \frac{d_{2} \epsilon_{x}}{\mu_{0} \mathrm{~b}_{x}} C_{1 D} \\
& \approx \frac{-1.1 \times 10^{-7}}{\tan \phi},
\end{aligned}
$$

where we used $C_{1 D}=-0.459$, and $b_{x} / \epsilon_{x}=\tan \phi$. The asymmetry can be set experimentally by choosing the angle $\phi$. Here we just assume the value $\tan \phi=0.1$ which gives an asymmetry of $-1.1 \times 10^{-6}$.

A more important quantity for assessing the viability of H/D APV experiments is the counting time required under ideal conditions, i.e. assuming one can achieve the shot-noise limit, and that the transition rate is well above background. The signal count $S$ is proportional to $\left(A_{\mathrm{PNC}}^{*} A_{\mathrm{PC}}+\right.$ c.c. $)$ times the factor $\eta J T$, where $\eta$ is the detection efficiency for the $\beta_{-1 / 2}$ state, $J$ is the metastable beam intensity per hyperfine component (atoms/s), and $T$ is the total counting time. The noise count $N$ is proportional to the square root of $\eta J T\left|\mathrm{~A}_{\mathrm{PC}}\right|^{2}$. Using Eqs. 13, 16 and 17 we find:

$$
\frac{S}{N}=4\left|\overline{\mathcal{V}}_{\mathrm{w}} C_{1 D}\right| \frac{1-\exp \left(-\gamma_{\alpha_{+1 / 2}} \tau / 2\right)}{\left(\gamma_{\alpha_{+1 / 2}} \gamma_{2 p}\right)^{1 / 2}}(\eta J T)^{1 / 2} .
$$


Note that Eqs. 20 does not depend on $\phi$ and, in the approximation we use here, the counting time is independent of the asymmetry chosen for the experiment. In fact, as pointed out by Hinds [54], if the phase between $A_{P C}$ and $A_{P N C}$ is chosen to maximize the interference term, the signal-to-noise is independent of $A_{P C}$.

Equation 20 has a maximum with respect $\gamma_{\alpha_{+1 / 2}}$ given by the condition: [54]:

$$
\gamma_{\alpha_{+1 / 2}}=2.5 / \tau
$$

which, for a given $\tau$, can be satisfied by adjusting the microwave power. Since the required power is readily obtained for the experiments considered here, we use Eq. 21 to eliminate $\gamma_{\alpha_{+1 / 2}}$ from Eq. 20 with the result:

$$
\left(\frac{S}{N}\right)_{o p t}=1.8\left|\overline{\mathcal{V}}_{\mathrm{w}} C_{1 D}\right|\left(\eta J T \tau / \gamma_{2 p}\right)^{1 / 2}
$$

The definitions of the parameters in this equation are summarized in Table 3 , Eq. 22 indicates that, apart from maximizing the beam intensity and detection efficiency, making $\tau$ as large as possible is the key requirement for maximizing $\mathrm{S} / \mathrm{N}$. This means an experiment based on a slow beam and a long interaction region. A hidden assumption here is that the linewidth of the $\alpha_{+1 / 2} \rightarrow \beta_{-1 / 2}$ transition is of order the width of the $\alpha_{+1 / 2}$ state (Eq. 17). In particular, the magnetic field must be constant and homogeneous at the level:

$$
\frac{\Delta B}{B}<\frac{\gamma_{\alpha_{+1 / 2}}}{\omega_{\alpha_{+1 / 2} \beta_{-1 / 2}}} \sim \frac{10^{-10} s}{\tau} .
$$

Eq. 22 also applies to measurements performed at the other level crossings listed in Table 2, providing the combinations of coupling constants listed there are substituted in place of $C_{1 D}$. We can use the optimized signal-to-noise ratio to estimate the minimum limits that can be placed on the electron-nucleon, weak-neutral-current coupling constants from experiments in $\mathrm{H}$ and $\mathrm{D}$. To do this, we assume that the counting time $T$ should not be more than about 5 days; with the understanding that, in reality, considerably more counting time would be required because of backgrounds and the need to study systematic errors. Then, taking the overall detection efficiency to be $\eta=0.5$, we plot the percent errors in the four constants as a function of the metastable beam intensity per hyperfine component $J$, and the time of passage of an atom through the interaction region $\tau$. We also give the magnetic field homogeneity required. The results for the deuterium couplings are given in Fig. 6, and those for hydrogen are given in Fig. 7 , For example, Fig. 6] indicates that to measure $C_{1 D}$ to $\sim 0.3 \%$ with an interaction time $\tau \sim 100 \mu \mathrm{s}$, requires a beam intensity of at least $J \simeq 5 \times 10^{14} \mathrm{~s}^{-1}$ and $\Delta B / B \sim 10^{-6}$. For longer interaction times $\tau$, the requirement on the beam intensity can be reduced but this also leads to more stringent specifications on the B-field homogeneity. The same experimental conditions would provide a measurement of $C_{2 D}$ to better than $20 \%$ and (based on Fig. 7) a $\sim 4 \%$ measurement of $C_{1 p}$. "Interesting" experiments can be done with smaller beam fluxes as can be seen from the lines in Figs. 6 and 7 indicating the model-independent limits on the four coupling constants as well as the "dot-dashed" 


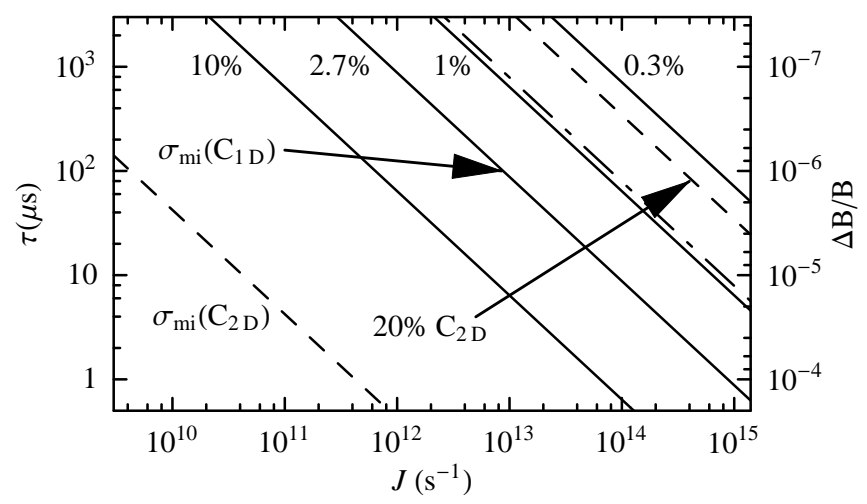

Figure 6. Deuterium APV sensitivity. Solid lines indicate projected minimum experimental uncertainties in $C_{1 D}$ (in percent of the SM value) for a generic APV experiment in D based on Eq. 22, These are plotted as a function of the time an atom spends in the interaction region $\tau$, and the beam intensity per hyperfine component $J$. The values assumed for the other parameters in Eq. 22 are summarized in Table 3 . The dashed lines are the projected uncertainties for a $C_{2 D}$ measurement at the $\beta \mathrm{e}$ crossing. The dot-dash line represents the uncertainty needed in a measurement of $C_{1 D}$ to determine $\sin ^{2} \theta_{w}$ with a precision equal to the current APV limit of $0.9 \%$. The lines marked $\sigma_{\mathrm{mi}}\left(C_{2 D}\right)$ and $\sigma_{\mathrm{mi}}\left(C_{1 D}\right)$ represent the uncertainties in these parameters based on a model-independent-fit to all neutral current data 1]. The axis on the right gives the magnetic field homogeneity $\Delta B / B$ based on Eq. 23 ,

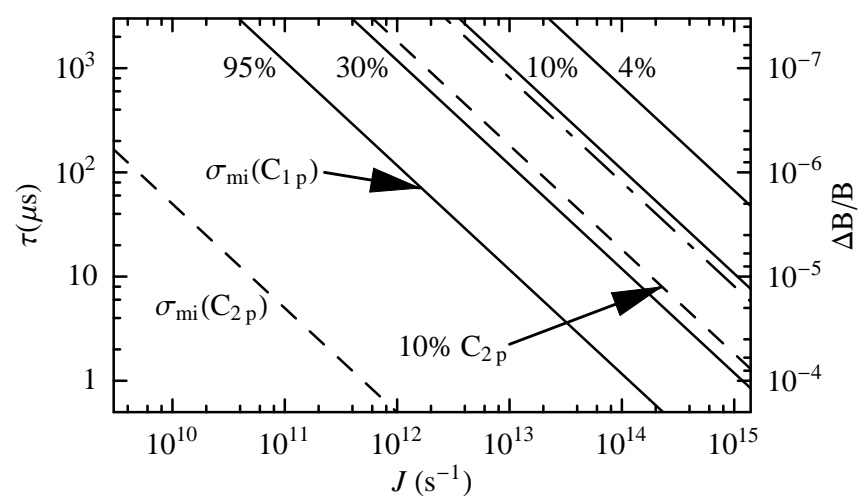

Figure 7. Hydrogen APV sensitivity. Projected minimum experimental uncertainties (as a percentage of the SM values) in the hydrogen weak interaction coupling constants based on Eq. 22, and plotted as a function of $\tau$ and $J$. (See caption to Fig. 6]) The solid lines refer to a measurement of $C_{1 p}$ and the dashed lines refer to measurement of $C_{2 p}$ at the $\beta \mathrm{e}$ crossing. The dot-dashed line corresponds to the uncertainty in $C_{1 p}$ required to match the current APV limit on $\sin ^{2} \theta_{w}$. The lines marked $\sigma_{\mathrm{mi}}\left(C_{1 p}\right)$ and $\sigma_{\mathrm{mi}}\left(C_{2 p}\right)$ correspond to the uncertainties in these parameters determined by the model-independent-fit discussed in Ref. [1]. 
lines which show the experimental uncertainty required to match the current APV limit on determination of $\sin ^{2} \theta_{w}$.

\section{Systematic Effects}

The $2^{2} \mathrm{~S}_{1 / 2}$ and $2^{2} \mathrm{P}_{1 / 2}$ levels in hydrogen can be mixed by unwanted electric fields and this can mimic parity mixing. The closeness of the levels enhances the PNC effects but it also increases the sensitivity of the experiments to systematic effects due to unwanted electric fields. In this section, we will consider two of the most troublesome effects expected in the experiment discussed in Section 5.

A number of essential elements of the treatment of systematic errors will not be covered here, however. These include the techniques for accurately aligning the apparatus, and reversing the vectors in the interaction region including: the polarization $\phi$, the microwave propagation $\mathbf{k}$, and the magnetic field $\mathbf{B}$. We will assume that the apparatus can be aligned to the levels achieved in the former experiments e.g. [44. However, in Appendix C a scheme for continuously monitoring the microwave polarization is discussed, that could make this reversal exact to the level of statistical accuracy. Another crucial issue concerns effects caused by variations in the intensity or direction of the fields as the atoms move through the apparatus [48]. These effects will not be discussed here. They are addressed in detail by e.g. Lévi and Williams [41.

To understand the problem of unwanted electric fields, we introduce electric fields $E_{x}^{u}, E_{y}^{u}$ and $E_{z}^{u}$ into our amplitudes. We also allow a misalignment of the microwave field which gives components $\epsilon_{y}^{m}$ and $\mathrm{b}_{y}^{m}$ in addition to the desired fields. The magnetic field defines the $z$ axis and we are free to take the microwave propagation vector $\mathbf{k}$ to lie in the $y-z$ plane. With these definitions, after adding the most important electricfield-induced terms, $\Lambda$ becomes 55 .

$$
\begin{aligned}
2 \hbar \Lambda=-\left(\mathrm{b}_{x}+\right. & \left.\mathrm{ib}{ }_{y}^{\mathrm{m}}\right) \mu_{0} \cos \Theta-\mathrm{i}\left(\epsilon_{x}+\mathrm{i} \epsilon_{y}^{m}\right) d_{W} \Delta_{\beta_{-1 / 2} f_{-1 / 2}} \\
& +\left(E_{x}^{u}+\mathrm{i} E_{y}^{u}\right) \epsilon_{z} d_{2}^{2} \Delta_{\beta_{-1 / 2} e_{+1 / 2}}-E_{z}^{u}\left(\epsilon_{x}+\mathrm{i} \epsilon_{y}^{m}\right) d_{2}^{2} \Delta_{\beta_{-1 / 2} f_{-1 / 2}} .
\end{aligned}
$$

Here, $d_{W}=\overline{\mathcal{V}} C_{1 D} d_{2}$. Under a polarization reversal $\phi \rightarrow-\phi$, the microwave components $\epsilon_{z}, \epsilon_{y}^{m}$, and $\mathrm{b}_{x}$ change sign while $\epsilon_{x}, \mathrm{~b}_{y}^{m}$, and $\mathrm{b}_{z}$ do not.

The first two terms in Eq. 24 (with $\epsilon_{y}^{m}=b_{y}^{m}=0$ ) are the amplitudes $\mathrm{A}_{\mathrm{PC}}$ and $\mathrm{A}_{\mathrm{PNC}}$ discussed in the previous section and the last two terms are the electric field induced amplitudes which are of concern in this section. The first point is that interferences among the electric field induced terms (E1E1 interferences) are relatively small since there are no applied electric fields. Also, these terms are readily eliminated because they are even in the reversal of $\mathbf{k}$, whereas the E1M1 PNC pseudoscalar term is odd under this reversal. Of the $E 1 M 1$ interferences, those that change sign under a reversal of the polarization $\phi$ are of most concern. The two most significant effects are: (1) a term caused by a transverse electric field which we will call the motional field effect, and, (2) a term caused by an electric field along $\mathbf{B}$ which we will call the stray $E_{z}$ systematic effect. In the following we estimate the size of these effects. Based on Eq. 21 and Eq. 17 
and taking $\tau=300 \mu$ s and $\tan \phi=0.1$, we find:

$$
\epsilon_{x} \rightarrow 0.16 \mathrm{~V} / \mathrm{cm}, \epsilon_{z} \rightarrow 0.016 \mathrm{~V} / \mathrm{cm}, b_{x} \rightarrow 5 \times 10^{-5} \mathrm{G} .
$$

\subsection{Motional electric field systematic effect}

The third term in Eq. 24 arises from mixing of $\beta_{-1 / 2}$ with $e_{+1 / 2}$ by a transverse electric field and, unlike the PNC term ( $2^{\text {nd }}$ term in Eq. 24) it is not resonant at the $\beta f$ crossing. The largest term is proportional to the combination of fields, $\epsilon_{z} \mathrm{~b}_{y}^{m} E_{y}^{u}$. This involves an electric field transverse to the magnetic field. The most significant field of this kind arises from the $\boldsymbol{v} \times \boldsymbol{B}$ "motional" electric field.

It is of interest to form the ratio $R^{\text {mot }}$ of the interference term arising from the motional electric field to that of the pseudoscalar interference term. This is:

$$
R^{m o t}=8 \times 10^{5} \frac{\left\langle E^{m o t}\right\rangle \sin \chi}{C_{1 D}}
$$

where $\chi$ is the microwave misalignment angle defined by $\mathrm{b}_{y}^{m}=\mathrm{b}_{x} \sin \chi$, and $\left\langle E^{\text {mot }}\right\rangle$ is the motional field (in Volts/cm) averaged over the beam velocity distribution. If the transverse velocity distribution were cylindrically symmetric and the axis of symmetry exactly coincident with a homogeneous magnetic field, the motional field systematic would vanish. For simplicity, we represent the imperfections of the real apparatus by a misalignment between the beam velocity and the magnetic field. Based on Fehrenbach's experiment 44] we take this angle to be $0.1 \mathrm{mrad}$. For a slow $(77 \mathrm{~K})$ deuterium beam, and a magnetic field of $\sim 1200 \mathrm{G}$, we find $\left\langle E^{m o t}\right\rangle \sim 10^{-4} \mathrm{~V} / \mathrm{cm}$. Assuming the microwave misalignment angle $\sin \chi=10^{-4}$, we have:

$$
R^{m o t} \approx \frac{0.01}{C_{1 D}} \approx 0.02
$$

The motional field effect can be distinguished from the pseudoscalar term because it is sensitive to slight changes in the direction of the B-field and it is non-resonant at the crossing.

For a new deuterium APV experiment, it would be desirable to improve the symmetry and alignment of the beam over what has been achieved in the past in order to suppress this effect by another order of magnitude so that it is smaller than $0.3 \%$ of the pseudoscalar term. But, regardless of the level of suppression obtained, this effect can be accurately measured and a correction can be applied to the final result of the experiment. To do this, the experiment must incorporate a continuous monitoring of the motional electric fields while the APV data are being taken. The general method for doing this is well known, as it is an essential technique in APV experiments. The unwanted fields are monitored by using the atoms themselves [37, 56]. In the present case, the motional field could be monitored by, e.g. periodically moving the magnetic field off the center of the level crossing (retuning the microwave frequency to an adjacent resonator mode). Away from the crossing, $A_{P N C}$ is suppressed, so the signal is dominated by the motional electric field effect. The limit on the motional field effect is determined by the statistical 
accuracy of the on-line monitoring, which by the arguments of Section 5, again reduces to the need for an intense, slow metastable beam.

The largest systematic error in the measurement of Fehrenbach was caused by a motional electric field and it is natural to ask why this is less problematic here. The first point is that the velocity of the slow $\mathrm{D}$ beam is a factor of $\sim 300$ smaller than his fast beam so the motional field is smaller by the same factor. Also, his experiment was performed at the crossing of the $e$ and $\beta$ levels which are mixed by the (transverse) motional field, so for an experiment done at the $\beta f$ crossing there is an additional relative suppression of approximately 10. The net result is a suppression of a factor of approximately 3000 for the motional field effects in the experiment discussed in Section 5 , relative to those in Fehrenbach's experiment.

\subsection{Stray $E_{z}$ systematic effect}

The last term in Eq. 24 arises from mixing between the $\beta_{-1 / 2}$ and $f_{-1 / 2}$ states by an unwanted "stray" electric field parallel to the magnetic field. Such a field could arise from surfaces in the interaction region, charged particles in the beam, etc. We call the most significant term, proportional to the fields $\epsilon_{x} \mathrm{~b}_{x} E_{z}^{u}$, the stray $E_{z}$ systematic effect. The microwave field combination is the same as that for the pseudoscalar interference, but this term has a dispersive shape as a function of magnetic field. It goes to zero near the center of the $\beta_{-1 / 2} f_{-1 / 2}$ crossing, and has a maximum at $\omega_{\beta_{-1 / 2} f_{-1 / 2}} \sim \gamma_{2 p} / 2$. The exact magnetic field where the stray $E_{z}$ term vanishes depends on couplings with more distant levels and it can be calculated to high precision. Due to field inhomogeneities, frequency shifts, the finite linewidth of the transition, etc., one cannot completely zero the stray $E_{z}$ effect. Rather, we introduce a parameter $\delta_{\Delta B}$ which is the degree of suppression of this term from its maximum value obtained by setting the magnetic field to the proper value. There is an additional suppression of this term since it is odd under a reversal of $\boldsymbol{B}$, while the pseudoscalar is even in this reversal. We assign another factor $\xi_{B}$ to account for this suppression.

The ratio $R^{E_{z}}$ of the stray $E_{z}$ interference term to the pseudoscalar is found using the same microwave field strengths as previously:

$$
R^{E_{z}}=10^{8} \frac{\left\langle E_{z}^{s}\right\rangle \delta_{\Delta B} \xi_{B}}{C_{1 D}}
$$

$\left\langle E_{z}^{s}\right\rangle$ is the average electric field along $\mathrm{z}$ seen by the atoms, expressed in $\mathrm{V} / \mathrm{cm}$.

The field $\left\langle E_{z}^{s}\right\rangle$ can be minimized by careful design of the interaction region, but here we just assume the value achieved by Fehrenbach which was $\left\langle E_{z}\right\rangle \sim 1 \mathrm{mV} / \mathrm{cm}$. We take the suppression factors to be $\xi_{B}=\delta_{\Delta B}=10^{-4}$, based on his results. Using these values we find:

$$
R^{E_{z}} \approx \frac{10^{-3}}{C_{1 D}} \approx-2 \times 10^{-3} .
$$

where the coupling constant was taken from Table 2. This estimate is an order of magnitude smaller than the motional field effect, and is smaller than our goal of $0.3 \%$ 
uncertainty in a measurement of the pseudoscalar term. The stray $E_{z}$ systematic effect is readily measured while the APV data are being recorded by setting the magnetic field off the crossing and looking for a term which is polarization dependent, odd in the magnetic field, and switches sign when the field is tuned to the other side of the crossing.

\section{Summary}

The major argument for consideration of parity experiments in $\mathrm{H}$ and $\mathrm{D}$, that there is negligible atomic physics uncertainty in the interpretation of the results, remains compelling. Parity violation experiments in deuterium could play a role in looking for new physics by providing a low energy measurement of $\sin ^{2} \theta_{W}$ to confirm the SM prediction for the "running of $\sin ^{2} \theta_{W}$ ". A $0.3 \%$ measurement of $C_{1 D}$ would improve the limit on $\sin ^{2} \theta_{W}$ provided by APV experiments by a factor of three.

Hydrogen and deuterium APV experiments could also contribute to determination of the electron-nucleon weak neutral current coupling constants listed in Table 1. Only $\mathrm{C}_{1 D}$ is accurately constrained by current data. The uncertainty in $\mathrm{C}_{1 p}$ is about the size of the $\mathrm{SM}$ prediction, and $\mathrm{C}_{2 p}$ and $\mathrm{C}_{2 D}$ are completely undetermined. The values of the individual constants could be determined by measuring APV at several level crossings. An updated list of the SM predictions for the parity violating matrix elements at level crossings in $\mathrm{H}$ and $\mathrm{D}$ are given in Table 2.

In Section 5 we analyzed a generic deuterium APV experiment, and found that a $0.3 \%$ measurement of $\mathrm{C}_{1 D}$ would be possible if a slow metastable beam with an intensity of $\sim 5 \times 10^{14}$ deuterium atoms/s per hyperfine level were available. In Appendix B we discuss some ideas for obtaining such a beam.

In Section 6, we discussed two systematic effects and found that, although they provide a challenge for a deuterium APV experiment, they are not insurmountable obstacles. After designing the experiment to minimize the systematic effects due to unwanted electric fields as much as possible, the elimination of these effects is done by monitoring them during the APV data taking, using the atoms themselves to measure the fields. Thus, the limitation on the experimental sensitivity due these systematic effects is dependent on counting statistics, and, in this sense, also depends on the intensity of the metastable beam.

\section{Acknowledgments}

We are indebted to Zheng-Tian Lu for significant contributions and encouragement. We also thank D. H. Beck for many useful discussions. Work supported by The U.S. Department of Energy, Office of Nuclear Physics, under Contract no. DE-AC02$06 \mathrm{CH} 11357$ (RJH). 


\section{Appendix A. Metastable quenching from beam-beam collisions}

Metastable quenching from beam-beam collisions places a practical limit on the density of metastable $\mathrm{D}(2 \mathrm{~s})$ atoms in a beam suitable for a deuterium APV experiment. Forrey et al. [57] calculated the cross sections for ionization, excitation-transfer, and elastic scattering in collisions between metastable hydrogen $2 \mathrm{~s}$ atoms at thermal energies. The most important metastable destruction mechanism was found to be double excitation transfer to $\mathrm{H}(2 \mathrm{p})$ :

$$
H(2 s)+H(2 s) \rightarrow H(2 p)+H(2 p)
$$

for which they found a cross section $\sigma^{s p}=9 \times 10^{-12} \mathrm{~cm}^{2}$ at $E=4.1 \mathrm{meV}$, varying as $E^{-1 / 2}$ at higher energies.

If we require that the mean free path $\lambda_{m f p}$ of the $\mathrm{D}(2 \mathrm{~s})$ atoms be a meter, this puts an upper limit on the metastable density:

$$
\rho_{2 s}<\frac{1}{\sigma^{s p} \lambda_{m f p}} \approx 10^{9} \mathrm{~cm}^{-3}
$$

Then, for a beam with an average velocity of $10^{5} \mathrm{~cm} / \mathrm{s}$, the $\mathrm{D}(2 \mathrm{~s})$ flux density must be $<10^{14} \mathrm{~cm}^{-2} \mathrm{~s}^{-1}$. We can take this limit to apply to an individual hyperfine component since one can quench out all unwanted components immediately after formation of the metastable beam using a deuterium spin filter [58]. For a metastable beam of $5 \times 10^{14} s^{-1}$ per hyperfine component, the beam diameter has to be $>2.5 \mathrm{~cm}$.

The metastable beam can also be quenched from beam-beam collisions with $\mathrm{D}(1 \mathrm{~s})$ and $\mathrm{D}_{2}$. The cross section[59] is about $2 \times 10^{-14} \mathrm{~cm}^{2}$, so for a mean free path of $1 \mathrm{~m}$

at $10^{5} \mathrm{~cm} / \mathrm{s}$, the flux density must be $<5 \times 10^{16} \mathrm{~cm}^{-2} \mathrm{~s}^{-1}$. For a $2.5 \mathrm{~cm}$ diameter beam this corresponds to $2 \times 10^{17} \mathrm{D}(1 \mathrm{~s}) / \mathrm{s}$.

\section{Appendix B. Optical production of metastable hydrogen and deuterium beams}

One possibility for obtaining a slow metastable $\mathrm{D}(2 \mathrm{~s})$ beam with sufficient intensity for a deuterium APV experiment is to use optical pumping starting with an intense dissociated deuterium beam. One such beam was produced by Harvey [60]. In his scheme, a beam of hydrogen atoms in the ground state was pumped to $3 \mathrm{p}$ using a Lyman- $\beta$ discharge lamp. The $3 p$ level decays to the 2 s state about $12 \%$ of the time. This work produced a beam with a flux of only $2 \times 10^{6}$ metastable atoms/s because of the low intensity of Lyman- $\beta$ radiation from the lamp. The development of intense beams of UV radiation using FEL technology might provide a considerable improvement in this respect.

The photon absorption cross section 61 for the $1 \mathrm{~s} \rightarrow 3 \mathrm{p}$ assuming the radiation does not resolve the $3 \mathrm{p}$ fine structure is given by

$$
\sigma_{3 p}=\frac{3 \lambda^{2}}{2 \pi} \frac{\gamma_{3 p 1 s}}{\gamma_{\text {line }}}
$$


where $\lambda$ is the transition wavelength, $\gamma_{3 p 1 s}$ is the ground state decay rate for the $3 p$ level and $\gamma_{\text {line }}$ is the transition linewidth. We find:

$$
\sigma_{1 s 3 p} \approx \frac{1.3 \times 10^{-12}}{\gamma_{\text {line }}(G H z)} \mathrm{cm}^{2} .
$$

We are interested in the amount of UV power at $102.6 \mathrm{~nm}$ needed to produce a metastable $\mathrm{D}(2 \mathrm{~s})$ beam (in a single hyperfine component) of $5 \times 10^{14} \mathrm{D}(2 \mathrm{~s}) \mathrm{s}^{-1}$ to serve as a benchmark for FEL development. Assuming a ground state beam of $2 \times 10^{17} \mathrm{~s}^{-1}$ with a diameter of $2.5 \mathrm{~cm}$ and a UV beam with a $3 \mathrm{~cm}$ diameter and accounting for the $12 \%$ branching ratio for $3 \mathrm{p} \rightarrow 2 \mathrm{~s}$, we find the power per bandwidth required is 40 $\mathrm{mW} / \mathrm{GHz}$.

If intense Lyman- $\alpha$ radiation is available, metastable hydrogen can be obtained by a three step process; two photon pumping $1 \mathrm{~s} \rightarrow 2 \mathrm{p} \rightarrow 4 \mathrm{~d}$ followed by decay to $2 \mathrm{~s}$. Lyman$\alpha$ radiation drives the transition $1 \mathrm{~s} \rightarrow 2 \mathrm{p}$ which is followed by a $2 \mathrm{p} \rightarrow 4 \mathrm{~d}$ transition saturated by a visible laser. Atoms in $4 \mathrm{~d}$ have a $3 \%$ branch to the 2 s state 62 . This scheme is similar to that used by Young, et al. for production of metastable Kr atoms [63, 64]. Although the branching ratio for the $4 \mathrm{~d}$ state is not as favorable as for the $3 \mathrm{~d}$ state, the oscillator strength for the transition $1 \mathrm{~s} \rightarrow 2 \mathrm{p}\left(f_{a b}=0.416\right)$ is five times larger than for the $1 \mathrm{~s} \rightarrow 3 \mathrm{p}$ transition. Also, an FEL capable of producing several watts of Lyman- $\alpha$ radiation is under development at Jefferson Lab in Virginia[65].

Another possibility for production of a metastable $\mathrm{H}$ beam is based on the twophoton transition $1 \mathrm{~s} \rightarrow 2 \mathrm{~s}$ with $243 \mathrm{~nm}$ radiation [66, 67, 68, 69]. This is a weak process since it does not involve an intermediate resonance state, and requires high intensity radiation. The needed intensity is normally obtained by focussing the light to a small spot, but this is incompatible with the limitation on the density of the metastable hydrogen atoms discussed in Appendix A, which requires a photon spot size of at least $5 \mathrm{~cm}^{2}$. For this reason, two-photon excitation does not appear to be a viable option for production of the required metastable beam. This conclusion is also supported by existing experimental data [70, 71].

\section{Appendix C. Normalization Transition}

In the generic experiment discussed in Section 5, the psuedoscalar interference term is measured by picking out the part of the $\alpha_{+1 / 2} \rightarrow \beta_{-1 / 2}$ transition rate that changes sign under the polarization reversal $\phi \rightarrow-\phi$. Ideally, the microwave field $\mathrm{b}_{x}$ that drives the $\mathrm{PC}$ transition would have the same strength in each polarization state, so the PC part of the transition would average to zero over the course of the experiment. In practice, it is not possible to achieve this to sufficient accuracy, and several independent reversals are used [41]. However, it is possible to monitor the field strength in each polarization state during accumulation of APV data, and make this reversal exact within the statistical uncertainty of the monitor. One way to do this is by periodically switching on an auxiliary static electric field $\boldsymbol{E}^{N}$ perpendicular to B. This adds a term to Eq. 13 of the 
form:

$$
2 \hbar \Lambda_{N}=d_{2}^{2} \Delta_{\beta_{-1 / 2} e_{+1 / 2}}\left(E_{x}^{(N)}+\mathrm{i} E_{y}^{(N)}\right) \epsilon_{z}
$$

The field direction can be chosen so that the new term is nearly orthogonal to the PNC amplitude. $\Lambda_{N}$ is proportional to the microwave electric field component $\epsilon_{z}$ which is directly proportional to the microwave magnetic field component $\mathrm{b}_{x}$ that needs to be measured. (See Fig. 1). The field strength $E^{(N)}$ is chosen so that $\Lambda_{N}$ is much larger than $\Lambda$ (Eq. 13) so that, when $\boldsymbol{E}^{(N)}$ is on, $\Lambda_{N}$ dominates the $\alpha_{+1 / 2} \rightarrow \beta_{-1 / 2}$ transition rate, and the contribution of the PNC interference term is sufficiently small that it does not contribute to the measurement of the field strength.

\section{References}

[1] W.-M. Yao et al. Particle Data Group. J. Phys. G, 33:1, 2006.

[2] J. Erler, A. Kurylov, and M. J. Ramsey-Musolf. Weak charge of the proton and new physics. Phys Rev D, 68:016006, 2003.

[3] G. W. Bennett et al. Final report of the E821 muon anomalous magnetic moment measurement at BNL. Phys. Rev. D, 73:72003, 2006.

[4] NuTeV Collaboration: G. P. Zeller et al. Precise determination of the electroweak parameters in neutrino-nucleon scattering. Phys. Rev. Lett., 88:091802, 2002.

[5] SLAC E158 Collaboration: P. L. Anthony et al. Observation of parity nonconservation in Møller scattering. Phys. Rev. Lett., 92:181602, 2004.

[6] J. P. Miller, E. de Rafael, and B. L. Roberts. Muon g-2: experiment and theory. Rep. Prog. Phys., 70:795, 2007.

[7] Qweak Collaboration: R. Carlini et al. The Q(weak) experiment: A search for physics at the TeV scale via a measurment of the proton's weak charge. Jefferson Lab Proposal E-02-020, Renewal PR-05-008.

[8] G. R. Smith for the Qweak Collaboration. Qweak: A precision measurement of the protons's weak charge. Eur. Phys. J. A., 24S2:155, 2005.

[9] P. E. Reimer. Measuring $\sin ^{2} \theta_{\mathrm{w}}$ with parity violating deep inelastic scattering. In Proc. of the $5^{\text {th }}$ Int'l Workshop on Neutrino Factories and Superbeams, AIP Conf. Proc., volume 721, page 371. 2004.

[10] J. Arrington et al. $\vec{e} \mathrm{H}$ parity violating deep inelastic scattering at CEBAF $6 \mathrm{GeV}$. Jefferson Lab Proposal PR-05-007, (X. Zheng and P. E. Reimer, Spokespersons).

[11] A. Ferroglia, G. Ossola, and A. Sirlin. The electroweak form factor $\hat{\kappa}\left(q^{2}\right)$ and the running of $\sin ^{2} \hat{\theta}_{w}$. Eur. Phys. J. C, 34:165, 2004.

[12] J. Erler and M. J. Ramsey-Musolf. Weak mixing angle at low energies. Phys Rev D, 72:073003, 2005.

[13] C. S. Wood, S. C. Bennett, D. Cho, B. P. Masterson, J. L. Roberts, C. E. Tanner, and C. E. Wieman. Measurement of parity nonconservation and an anapole moment in cesium. Sci., 275:1759, 1997.

[14] N. H. Edwards, S. J. Phipp, P. E. G. Baird, and S. Nakayama. Precise measurement of parity nonconserving optical rotation in atomic thallium. Phys. Rev. Lett., 74:2654, 1995.

[15] P. A. Vetter, D. M. Meekhof, P. K. Majumder, S. K. Lamoreaux, and E. N. Fortson. Precise test of electroweak theory from a new measurement of parity nonconservation in atomic thallium. Phys. Rev. Lett., 74:2658, 1995.

[16] M. A. Bouchiat and C. C. Bouchiat. Weak neutral currents in atomic physics. Phys. Lett., 48B:111, 1974.

[17] G. Feinberg and M. Y. Chen. $2 \mathrm{~s}_{1 / 2} \rightarrow 1 s_{1 / 2}+$ one photon decay of muonic atoms and parityviolating neutral current interactions. Phys. Rev. D, 10:190, 1974. 
[18] G. Feinberg and M. Y. Chen. Hyperfine effects in parity-violating muonic-atom decays. Phys. Rev. D, 10:3789, 1974.

[19] This definition differs from that for $C_{2}$ (deuterium) given in Dunford, et al. Phys. Rev. A, 18:2421, 1978.

[20] E. D. Commins and P. H. Bucksbaum. The parity nonconserving electron-nucleon interaction. Ann. Rev. Nucl. Part. Sci., 30:1, 1980.

[21] P. Fayet. The Standard Model and beyond. In B. Frois and M.-A. Bouchiat, editors, Parity Violation in atoms and polarized electron scattering, page 3. World Scientific, Singapore, 1999.

[22] W. J. Marciano and A. Sirlin. Radiative corrections to atomic parity violation. Phys Rev D, $27: 552,1983$. W. J. Marciano and A. Sirlin. Some general properties of the $\mathrm{O}(\alpha)$ corrections to parity violations in atoms. Phys Rev D, 29:75, 1984.

[23] Note: the signs here differ from Ref. 22] in order to conform to the quark couplings defined in Ref. [1].

[24] R. N. Cahn and G. L. Kane. Parity violations in hydrogen and the fundamental structure of the weak current. Phys. Lett., 71B:348, 1977.

[25] J. Collins, F. Wilczek, and A. Zee. Low-energy manifestations of heavy particles: application to the neutral current. Phys Rev D, 18:242, 1978.

[26] I. B. Khriplovich, and R. V. Korkin. P and T odd electromagnetic moments of deuteron in chiral limit. Nucl. Phys. A, 665:365, 2000.

[27] B. A. Campbell, J. Ellis, and R. A. Flores. Parity violation in light atoms and the strange spin content of the proton. Phys. Lett. B, 225:419, 1989.

[28] R. D. Young, R. D. Carlini, A. W. Thomas and J. Roche. Testing the Standard Model by precision measurement of the weak charges of quarks. arXiv:0704.2618, 2007.

[29] The energy level diagrams in this paper are based on a precise theory of the Zeeman effect in L. P. Lévy, Ph.D. Dissertation, "Parity and time reversal invariance in the hydrogen atom", Univ. of Michigan, 1982.

[30] W. E. Lamb Jr. Fine structure of the hydrogen atom. III. Phys. Rev., 85:259, 1952.

[31] W. E. Lamb Jr. and R. C. Retherford. Fine structure of the hydrogen atom. I. Phys. Rev., 79:549, 1950.

[32] Ya. I. Azimov, A. A. Ansel'm, A. N. Moskalev, and R. M. Ryndin. Some parity-nonconservation effects in emission by hydrogenlike atoms. Sov. Phys. -JETP, 40:8, 1975.

[33] R. W. Dunford and R. R. Lewis. Analysis of weak neutral currents in hydrogenic ions. Phys. Rev. $A, 23: 10,1981$.

[34] R. R. Lewis and W. L. Williams. Parity nonconservation in the hydrogen atom. Phys. Lett., 59B:70, 1975.

[35] E. A. Hinds and V. W. Hughes. Parity nonconservation in hydrogen involving magnetic/electric resonance. Phys. Lett., 67B:487, 1977.

[36] R. W. Dunford, R. R. Lewis, and W. L. Williams. Parity nonconservation in the hydrogen atom. II. Phys Rev A, 18:2421, 1978.

[37] R. W. Dunford, Ph.D. Dissertation, "Parity nonconservation in the hydrogen atom", Univ. of Michigan, 1978.

[38] E. G. Adelberger et al. A technique for measuring parity non-conservation in hydrogenic atoms. Nucl. Instrum. Meth., 179:181, 1981.

[39] E. A. Hinds. Radiofrequency spectroscopy. In G. W. Series, editor, The spectrum of atomic hydrogen - advances, page 243. World Scientific, Singapore, 1988.

[40] L. P. Lévy and W. L. Williams. Experimental test of Laporte's rule in hydrogen. Phys. Rev. Lett., 48:607, 1982.

[41] L. P. Lévy and W. L. Williams. Parity nonconservation in the hydrogen atom. III. Phys. Rev. A, 30:220, 1984.

[42] L. P. Lévy and W. L. Williams. Role of damping and T invariance in induced transitions in $\mathrm{H}(2 \mathrm{~s})$. Phys. Rev. Lett., 48:1011, 1982. 
[43] R. W. Dunford, D. S. Gemmell, M. Jung, E. P. Kanter, H. G. Berry, A. E. Livingston, S. Cheng, and L. J. Curtis. E1-M1 damping interference in the electric field quenching of metastable Ar $^{17+}$. Phys. Rev. Lett., 79:3359, 1997.

[44] C. C. W. Fehrenbach, Ph.D. Dissertation, "An experimental limit on parity mixing in atomic hydrogen", Univ. of Michigan, 1993.

[45] T. E. Chupp, Ph.D. Dissertation, Parity nonconservation in the hydrogen atom, Univ. of Washington, 1983.

[46] I. B. Khriplovich. Parity Nonconservation in Atomic Phenomena. Gordon and Breach, Philadelphia, 1991.

[47] R. W. Dunford and Th. Stöhlker. PNC in hydrogen; different prospects using heliumlike ions. In B. Frois and M.-A. Bouchiat, editors, Parity Violation in atoms and polarized electron scattering, page 356. World Scientific, Singapore, 1999.

[48] R. T. Robiscoe. Parity nonconservation experiments in radio-frequency cavities. J. Appl. Phys., 53:3380, 1982.

[49] G. Schulten. Microwave optical ring resonators. IEEE Trans. on Microwave Theory and Techniques, 15:54, 1967. ieeexplore.ieee.org.

[50] L. N. Menegozzi and W. E. Lamb Jr. Theory of a ring laser. Phys. Rev. A, 8:2103, 1973.

[51] T. J. Balle and W. H. Flygare. Fabry-Perot cavity pulsed Fourier transform microwave spectrometer with a pulsed nozzle particle source. Rev. Sci. Instrum., 52:33, 1981.

[52] R. W. Dunford. Parity nonconservation in singly ionized helium. Phys Rev A, 33:2949, 1986.

[53] An overall factor of $\cos \Theta_{+} \cos \Theta_{-}$has been left out of Eq. 13. Here, $\Theta_{ \pm}$are hyperfine mixing parameters for deuterium [37, 36]. At the $\beta$ f crossing $\cos \Theta_{+} \simeq \cos \Theta_{-} \simeq 0.999$.

[54] E. A. Hinds. Sensitivity and the role of level crossings in measurements of parity nonconservation using metastable atoms. Phys. Rev. Lett., 44:374, 1980.

[55] For the purposes of this article, it is sufficient to include only the mixings of $\beta_{-1 / 2}$ with the $2 \mathrm{P}_{1 / 2}$ levels. We neglect small hyperfine induced mixings, perturbations of $\alpha_{+1 / 2}$ by electric fields, and mixings with more distant $\mathrm{p}$ states. All these effects need to be considered in a full treatment.

[56] S. L. Gilbert and C. E. Wieman. Atomic-beam measurement of parity nonconservation in cesium. Phys. Rev. A., 34:792, 1986.

[57] R. C. Forrey, R. Côté, A. Dalgarno, S. Jonsell, A. Saenz, and P. Froelich. Collisions between metastable hydrogen atoms at thermal energies. Phys Rev Lett., 85:4245-4248, 2000.

[58] J. L. McKibben, G. P. Lawrence, and G. G. Ohlsen. Nuclear spin filter. Phys. Rev. Lett., 20:1180, 1968.

[59] S. R. Ryan, S. J. Czuchlewski, and M. V. McCusker. Collisional quenching of metastable hydrogen atoms by atoms and molecules. Phys. Rev. A, 16:1892, 1977.

[60] K. C. Harvey. Slow metastable atomic hydrogen beam by optical pumping. J. Appl. Phys., 53:3383, 1982.

[61] D Budker, D. F. Kimball, and D. P. DeMille. Atomic Physics. Oxford University Press, Oxford, 2004.

[62] Another possibility would be to drive $2 p \rightarrow 4 s$ in the second step. The $4 s$ state has a larger branch to $2 \mathrm{~s}(5 \%)$, but the oscillator strength for this transition, is a factor of 40 smaller than that for $2 p \rightarrow 4 d$.

[63] L. Young, D. Yang, and R. W. Dunford. Optical production of metastable krypton. J. Phys. B, 35:2985-2992, 2002

[64] S.-M. Ding, A. M. Bailey, A. M. Davis, R. W. Dunford, Z.-T. Lu, T. P. O. Connor, and L. Young. A thermal beam of metastable krypton atoms produced by optical excitation. Rev. Sci. Instrum., 78:023103, 2007.

[65] G. P. Williams 2004 private communication.

[66] F. Bassani, J. J. Forney, and A. Quattropani. Choice of gauge in two-photon transitions: 1s-2s transition in atomic hydrogen. Phys. Rev. Lett., 39:1070, 1977.

[67] M. G. Boshier, P. E. G. Baird, C. J. Foot, E. A. Hinds, M. D. Plimmer, D. N. Stacey, J. B. Swan, 
D. A. Tate, D. M. Warrington, and G. K. Woodgate. Laser spectroscopy of the 1s-2s transition in hydrogen and deuterium: Determination of the 1s Lamb shift and the Rydberg constant. Phys. Rev. A, 40:6169, 1989.

[68] M. Dörr, O Latinne, and C. J. Joachain. Time evolution of two-photon population transfer between the 1s and 2s states of a hydrogen atom. Phys. Rev. A, 55:3697, 1997.

[69] L. P. Yatsenko, B. W. Shore, T. Halfmann, K Bergmann, and A Vardi. Source of metastable H(2s) atoms using the Stark chirped rapid-adiabatic-passage technique. Phys. Rev. A, 60:R4237, 1999.

[70] Zehuang Lu, L. B. Wang, E. Thorsland, D. Beck, J. White, and R. Holt. Generation of metastable atomic hydrogen beam by optical pumping for atomic parity violation measurement. Bull. Am. Phys. Soc., 46:51, 2001.

[71] D. H. Beck 2007 private communication. 